\title{
Astrophysical constraints and insights on extended relativistic gravity.
}

\author{
S. Mendozal ${ }^{\text {* }}$ and Gonzalo J. Olmo2 2 † \\ ${ }^{1}$ Instituto de Astronomía, Universidad Nacional Autónoma de México, AP 70-264, Distrito Federal 04510, México \\ 2 Departamento de Física Teórica and IFIC, Centro Mixto Universidad de \\ Valencia - CSIC. Universidad de Valencia, Burjassot-46100, Valencia, Spain
}

(Dated: June 19, 2018)

\begin{abstract}
We give precise details to support that observations of gravitational lensing at scales of individual, groups and clusters of galaxies can be understood in terms of non-Newtonian gravitational interactions with a relativistic structure compatible with the Einstein Equivalence Principle. This result is derived on very general grounds without knowing the underlying structure of the gravitational field equations. As such, any developed gravitational theory built to deal with these astrophysical scales needs to reproduce the obtained results of this article.
\end{abstract}

PACS numbers: 95.30.Sf,04.50.Kd,04.25.Nx,98.62.Dm,98.62.Sb

Keywords: Relativity and gravitation;Modified theories of gravity;Post-Newtonian approximation;Dynamics; Gravitational lenses

\section{INTRODUCTION}

The first solid step towards a full development of a nonrelativistic theory of gravity was made by Newton in his PhilosophiceNaturalis Principia Mathematica book [1]. The starting point of this non-relativistic theory of gravity began with the third law of planetary motion published by Kepler in his Harmonices Mundi book [2]. For the known 7 planets back then, this law represents a relation between the mass of the sun $M$, a planet's particular distance to the sun $r$ and the velocity $v$ of a planet about the sun: $v \propto(M / r)^{1 / 2}$, for circular orbits. The requirement of centripetal balance during the motion of planets yields:

$$
a=-v^{2} / r=-G M / r^{2},
$$

where the proportionality factor $G$ is Newton's gravitational constant and the minus sign appears because of the attractive nature of gravity. The acceleration $a$ produced by the sun on a test planet is thus given by a force inversely proportional to its separation from it and linearly depends on the sun's mass. The right hand side of equation (10) is the simplest form of the mathematical force of gravity introduced by Newton.

In recent years, through dynamical observations of galaxies [e.g [3, and references therein], dwarf spheroidal galaxies [cf. 4], globular clusters [5, 6] and even wide open binaries [7], it has became clear that Kepler's third law appears not to hold in its classical form on these systems, but rather requires a modification known as the Tully-Fisher law:

$$
v \propto M^{1 / 4}
$$

\footnotetext{
* Email address: sergio@astro.unam.mx

$\dagger$ Email address: gonzalo.olmo@csic.es
}

where $v$ represents the velocity (or dispersion velocity for a dynamically pressure supported astrophysical system) and $M$ is the mass (could be internal mass within a radius r) of the system. Similarly to Newton's approach, the requirement of centripetal balance means that the acceleration $a \propto v^{2} / r$ at a distance $r$ from the configuration's centre and so

$$
a=-G_{\mathrm{M}} \frac{M^{1 / 2}}{r},
$$

where the constant of proportionality has been written as $G_{\mathrm{M}}$ and the minus sign has been introduced in order to manifest the attractive nature of the gravitational force. Equation (3) can be seen as a motivation to suspect that a new theory of gravity needs to be developed in these astrophysical systems, since its right hand side represents a relation between the acceleration felt by a test body of mass $M$ at a distance $r$. In this sense, the proportionality constant $G_{\mathrm{M}}$ can be seen as a new gravitational constant, with dimensions of squared length over squared time by the square root of mass.

This means that, in the same way as $G$ is regarded as a fundamental constant of nature, $G_{\mathrm{M}}$ should aspire to the same privileged status. However, in order to gain merits in that direction, $G_{\mathrm{M}}$ should play an essential role in the description of relativistic phenomena on its corresponding scales. Nonetheless, it should be noted that the construction of equations (11) and (31) are completely independent, since they both depend on different and unrelated data sets. As such, the constants $G$ and $G_{\mathrm{M}}$ can safely be postulated as independent. Given this independence, one is allowed to think of both as equally fundamental.

Requiring gravity to be described by equation (11) at some particular scales and behaving at some others according to relation (3), means that the scale invariance of gravity is necessarily broken. One can postulate that at some astrophysical scales gravity is Newtonian and requires modification at some others. The scale is not 
just a "fixed" distance scale. From the experimental astronomical evidence mentioned above it follows that the modified regime of gravity appears when the ratio of the mass of a given astrophysical system divided by its characteristic radius is sufficiently small as compared to the corresponding solar system value, which suggests that the transition scale is dynamical rather a simple fixed length. A given test particle sufficiently far away from a mass distribution is thus in this modified regime of gravity.

The approach introduced above for the description of gravitational phenomena departing from standard Newtonian gravity can be connected with the simplest version of the Modified Newtonian Dynamics (MOND) formula by replacing the constant $G_{\mathrm{M}}$ with a new constant $a_{0}$ introduced by Milgrom [8] with dimensions of acceleration through the relation:

$$
a_{0}:=G_{\mathrm{M}}^{2} / G
$$

and so equation (3) can be written as:

$$
a=-\frac{\left(a_{0} G M\right)^{1 / 2}}{r} .
$$

Since Milgrom's acceleration constant $a_{0} \approx 1.2 \times$ $10^{-10} \mathrm{~m} \mathrm{~s}^{-2}[3]$ it follows that:

$$
G_{\mathrm{M}} \approx 8.94 \times 10^{-11} \mathrm{~m}^{2} \mathrm{~s}^{-2} \mathrm{~kg}^{-1 / 2} .
$$

In this regard it is quite important to notice that the formulation of Milgrom [8] describes a modification on the dynamical sector of Newton's second law and not on the particular form of the gravitational force [cf. 9]. This is quite evident from the initial development of the theory, in which the requirement that the squared of the acceleration $a^{2}$ proportional to the Newtonian acceleration $G M / r^{2}$ implies flattening of rotation curves in spiral galaxies. In this relation, the proportionality constant $a_{0}$ with dimensions of acceleration, is required to be a fundamental quantity of nature. By doing so, the TullyFisher law is obtained as a consequence of the proposed modification of dynamics.

With the approach made here, it follows that the TullyFisher law forces the construction of a full gravitational theory in systems where Newtonian gravity does not work. In its simplest form, the developed theory must converge to equation (3). As such, no need for modification of Newton's second law needs to be introduced, since only a non-scale invariant character for the gravitational law is directly inferred from the observational data.

In our view, the introduction of $G_{\mathrm{M}}$ as a fundamental constant of gravity, rather than $a_{0}$ as a new fundamental acceleration scale, sheds light onto the strategy to follow to unveil the structure of the underlying theory. In fact, $G_{\mathrm{M}}$ points towards a modification on the gravitational sector, whereas $a_{0}$ could point towards a break down or possible extensions of special relativity (due to the existence of a universal acceleration scale, similarly as with the speed of light), with potentially dramatic implications even in non-gravitational systems.

If Newtonian gravity breaks at a certain scale, one can legitimately wonder whether the relativistic structure of gravitational interactions remains valid or may require a full reformulation. To explore these aspects one should study not only the dynamics of slow massive particles (e.g. equation (3) ) but also the motion of relativistic particles (such as photons) in astrophysical scenarios probing the gravitational field in this new regime. Being conservative, one may assume that Einstein's insights on the geometrical interpretation of gravity remain valid in this regime. As such, it is perfectly reasonable to assume that the Einstein Equivalence Principle remains valid, which implies that test particles satisfy the geodesic equation:

$$
\frac{\mathrm{d}^{2} x^{\alpha}}{\mathrm{d} s^{2}}+\Gamma_{\mu \nu}^{\alpha} \frac{\mathrm{d} x^{\mu}}{\mathrm{d} s} \frac{\mathrm{d} x^{\nu}}{\mathrm{d} s}=0
$$

where $\Gamma_{\beta \eta}^{\alpha}$ are the Christoffel symbols and summation convention is used over repeated indices (Greek indices vary from 0 to 3 and Latin ones from 1 to 3 ). The coordinates $x^{\alpha}=(c t, x, y, z)$ and the interval $\mathrm{d} s^{2}=g_{\mu \nu} \mathrm{d} x^{\mu} \mathrm{d} x^{\nu}$ for a metric tensor $g_{\mu \nu}$ and a velocity of light $c$.

By knowing Tully-Fisher's modification of Kepler's third law (2) and the geodesic equation (7), then at second order perturbation, the bending of light is completely determined up to a constant [10]. This is due to the fact that to this order of approximation the motion of photons only depends on the non-relativistic gravitational potential and a parameter $\gamma=$ const., which measures the proportionality between the leading (second) order corrections of the time and spatial metric components in isotropic coordinates.

We show in this article that experimental data from astronomical observations point us to show that only modifications of Kepler's third law are necessary in order to reproduce observations of gravitational lensing. In this article we postulate the Einstein Equivalence Principle to be valid and combine it with the non-relativistic gravitational potential associated to the modified Kepler's third law (Tully-Fisher law). This approach only assumes that gravitation is a geometric phenomenon and as such, we do not need to know the underlying set of relativistic field equations to find that the corresponding predictions for the bending of light are compatible with the observations of galaxies and groups of galaxies. This approach is parallel to the strategy followed to understand the relativistic behaviour of gravity in the solar system, where traditional Kepler's third law holds.

Additionally, we interpret the results by Hernandez, Jiménez, and Allen [7] on the failure of Kepler's third law for wide binary systems, as a way to test a key aspect of the mathematical structure of the underlying theory of gravity, namely whether or not external boundary conditions influence the internal dynamics of local grav- 
itational systems, which is sometimes referred to as an external field effect [3]. This effect means that for example, a gravitating system in the modified Keplerian regime embedded on an external standard Newtonian (or Keplerian) field, would behave in a Newtonian way. Hernandez, Jiménez, and Allen [7] studied orbits of wide binary stars $\sim 1 M_{\odot}$ separated by $\gtrsim 7000 \mathrm{AU}$. These bound objects are embedded in our galaxy and are subject to its Newtonian gravity. As such, if an external field effect occurs, then these objects would orbit each other in a standard way, following Kepler's third law. However, their analysis shows that a violation of Kepler's third law occurs in these systems. The large statistics and precise astrometry to be obtained with the GAIA probe of the European Space Agency in the near future, should provide a strong test for the validity of Kepler's third law at scales yet to be explored. Furthermore, we show that lensing observations strongly support the validity of equation (7), implying that the effects of external gravitational fields can be removed by a suitable choice of local coordinates (a freely falling frame). To the light of these results, the idea of an external field effect appears as an artificial construction (possibly related to the specific mathematical realisations of the theory).

The article is organised as follows. In section [I] we discuss the simplest properties that an extended theory of gravity must obey on systems where the Tully-Fisher law is valid. In section [II] we use gravitational lensing observations in individual, groups and clusters of galaxies combined with the Tully-Fisher law to obtain empirical relations for the metric coefficients of a spherically symmetric space-time at second perturbation order. Finally in section IV we summarise our main results and discuss them in lights of future theoretical developments in the search for a complete extended theory of gravity not requiring the use of dark matter in the description of astrophysical phenomena

\section{BASIC PROPERTIES FOR A RELATIVISTIC EXTENSIONS OF THE TULLY-FISHER LAW}

In order to seek for a complete set of fundamental observables of the underlying relativistic theory of gravity in the regime where Kepler's third law requires modification in the form of a Tully-Fisher law, let us analyse the behaviour of gravity in the weak field limit, having in mind the behaviour of massive particles and photons.

Consider a fixed point mass $M$ at the centre of coordinates generating a gravitational field. The underlying space-time is thus static and its spherically symmetric metric line element $\mathrm{d} s$ can be written in spherical Schwarzschild coordinates as:

$$
\mathrm{d} s^{2}=g_{\mu \nu} \mathrm{d} x^{\mu} \mathrm{d} x^{\nu}=g_{00} c^{2} \mathrm{~d} t^{2}+g_{11} \mathrm{~d} r^{2}-r^{2} \mathrm{~d} \Omega^{2} .
$$

In the previous equation and in what follows the spacetime coordinates $\left(x^{0}, x^{1}, x^{2}, x^{3}\right)=(c t, r, \theta, \varphi)$, where $t$ represents time, $r$ the radial coordinate and the polar and azimuthal angles are given by $\theta$ and $\varphi$ respectively. The angular displacement $\mathrm{d} \Omega^{2}:=\mathrm{d} \theta^{2}+\sin ^{2} \theta \mathrm{d} \varphi^{2}$. The symmetry of the problem means that the unknown metric components $g_{00}$ and $g_{11}$ are functions that depend on the radial coordinate $r$ only. In the limit where the speed of light $c \rightarrow \infty$, the radial component of the geodesic equation (77) in these coordinates is given by:

$$
\frac{1}{c^{2}} \frac{\mathrm{d}^{2} r}{\mathrm{~d} t^{2}}=\frac{1}{2} g^{11} \frac{\partial g_{00}}{\partial r} .
$$

The previous relation holds since we have used the following approximation for the weak field limit: $\mathrm{d} s=c \mathrm{~d} t$, and so, due to the fact that the velocity $v \ll c$ then $v^{i} \ll \mathrm{d} x^{0} / \mathrm{d} t$ with $v^{i}:=(\mathrm{d} r / \mathrm{d} t, r \mathrm{~d} \theta / \mathrm{d} t, r \sin \theta \mathrm{d} \varphi / \mathrm{d} t)$.

The lowest perturbation order of equation (9) is obtained when its left-hand side is of order $v^{2} / c^{2}$ and when its right-hand side is of order $g_{00}=1+\phi / c^{2}$ [10]. Both are orders $\mathcal{O}\left(1 / c^{2}\right)$ of the underlying theory, or simply $\mathcal{O}(2)$.

In this weak-field slow-motion approximation, a particle bounded to a circular orbit about the mass $M$ experiences a centrifugal radial acceleration given by:

$$
\frac{\mathrm{d}^{2} r}{\mathrm{~d} t^{2}}=\frac{v^{2}}{r}
$$

for a circular tangential velocity $v$.

The motion of material and light particles at this lowest perturbation order is such that the metric components are given by [10, 11]:

$$
\begin{aligned}
& g_{00}={ }^{(0)} g_{00}+{ }^{(2)} g_{00}+\mathcal{O}(4)=1+{ }^{(2)} g_{00}+\mathcal{O}(4), \\
& g_{11}={ }^{(0)} g_{11}+{ }^{(2)} g_{11}+\mathcal{O}(4)=-1+{ }^{(2)} g_{11}+\mathcal{O}(4), \\
& g_{22}=-r^{2}, \\
& g_{33}=-r^{2} \sin ^{2} \theta,
\end{aligned}
$$

where the superscript $(p)$ denotes the order $\mathcal{O}(p)$ at which a particular quantity is approximated. The nonrelativistic potential $\phi$ is defined as [e.g 10, 12, 13]:

$$
{ }^{(2)} g_{00}=\frac{2 \phi}{c^{2}} \text {. }
$$

From equations (11) it follows that the contravariant metric components are given by:

$$
\begin{aligned}
& g^{00}={ }^{(0)} g^{00}+{ }^{(2)} g^{00}+\mathcal{O}(4)=1-{ }^{(2)} g_{00}+\mathcal{O}(4), \\
& g^{11}={ }^{(0)} g^{11}+{ }^{(2)} g^{11}+\mathcal{O}(4)=-1-{ }^{(2)} g_{11}+\mathcal{O}(4), \\
& g^{22}=-1 / r^{2} \\
& g^{33}=-1 / r^{2} \sin ^{2} \theta
\end{aligned}
$$

At this level of approximation, the motion of nonrelativistic massive particles only requires knowledge of the metric component ${ }^{(2)} g_{00}$. The motion of photons is fully determined by additionally knowing ${ }^{(2)} g_{11}$ [cf. 10] 


\section{TULLY-FISHER'S RELATIVISTIC CORRECTIONS}

Let us take the radial component (9) of the geodesic equations (7) at the lowest relativistic perturbation order. In this limit, the rotation curve for test particles bound to a circular orbit about a mass $M$ with circular velocity $v$ is given by equation (10) and so:

$$
\frac{v^{2}}{c^{2} r}=\frac{1}{2} \frac{\partial^{(2)} g_{00}}{\partial r}
$$

Since we are interested in the behaviour of particles where the modified Kepler's third law (or Tully-Fisher law) holds, equation (2) can be written as:

$$
v=G_{\mathrm{M}}^{1 / 2} M^{1 / 4}
$$

Substitution of this equation on relation (14) yields:

$$
\frac{\partial^{(2)} g_{00}}{\partial r}=-\frac{2}{r}\left(\frac{v}{c}\right)^{2}=-\frac{2 G_{\mathrm{M}} M^{1 / 2}}{c^{2} r},
$$

which has a direct analytical solution:

$$
{ }^{(2)} g_{00}=-2\left(\frac{v}{c}\right)^{2} \ln \left(\frac{r}{r_{\star}}\right)=-\frac{2 G_{\mathrm{M}} M^{1 / 2}}{c^{2}} \ln \left(\frac{r}{r_{\star}}\right) \text {, }
$$

where $r_{\star}$ is an arbitrary length.

Having obtained the ${ }^{(2)} g_{00}$ component, which determines the non-relativistic motion of massive particles, we now proceed to obtain the ${ }^{(2)} g_{11}$ component. In the literature it is customary to define a new scalar potential $\psi$ as:

$$
{ }^{(2)} g_{11}=-\frac{2 \psi}{c^{2}}
$$

in complete analogy with equation (12). The introduction of this potential can be justified considering a more general scenario. Without requiring spherical symmetry, the spatial part of the metric can be written as $g_{i k} \mathrm{~d} x^{i} \mathrm{~d} x^{k}$, with ${ }^{(0)} g_{k l}=\delta_{k l}$ being the Minkowskian part. The second order perturbation corrections of $g_{k l}$ could in principle involve other potentials (and not only $\phi$ or $\psi$ ). By a suitable choice of coordinates, one can get rid of the anisotropic contributions at the same perturbation order, which turns $g_{k l}$ into a diagonal form. Given the isotropy of space, there is no preferred direction and so ${ }^{(2)} g_{i k} \propto \delta_{i k}$. It is natural to expect that the leading order $\mathcal{O}(2)$ correction must be of the same order of magnitude as the gravitational potential $\phi$. Accordingly $g_{k l}=\left(1+2 \gamma \phi / c^{2}\right) \delta_{k l}$, where $\gamma$ is a proportionality constant, and so

$$
\mathrm{d} s^{2}=g_{00} \mathrm{~d} t-\left(1+2 \gamma \phi / c^{2}\right) \delta_{k l} \mathrm{~d} x^{k} \mathrm{~d} x^{l} .
$$

Since spherical Schwarzschild coordinates are widely used in astrophysical literature, let us calculate the metric component $g_{11}$ in such coordinates. The conversion is straightforward since

$$
g_{11} \mathrm{~d} r^{2}+r^{2} \mathrm{~d} \Omega^{2}=\left(1+2 \gamma \phi / c^{2}\right)\left(\mathrm{d} \tilde{r}^{2}+\tilde{r}^{2} \mathrm{~d} \Omega^{2}\right),
$$

for spherical isotropic coordinates $(c t, \tilde{r}, \theta, \varphi)$. Using equations (17) and (12) it follows that

$$
r=\tilde{r}\left[1-\gamma\left(\frac{G_{\mathrm{M}} M^{1 / 2}}{c^{2}}\right) \ln \left(\frac{r}{r_{\star}}\right)\right]
$$

and so,

$$
\mathrm{d} r=\mathrm{d} \tilde{r}\left[1-\frac{G_{\mathrm{M}} M^{1 / 2}}{c^{2}} \ln \left(\frac{\tilde{r}}{r_{\star}}\right)-\frac{G_{\mathrm{M}} M^{1 / 2}}{c^{2}}\right],
$$

at perturbation order $\mathcal{O}(2)$. This means that:

$$
\psi=-\gamma G_{\mathrm{M}} M^{1 / 2}
$$

which yields:

$$
{ }^{(2)} g_{11}=-\frac{2 \gamma G_{\mathrm{M}} M^{1 / 2}}{c^{2}} \text {. }
$$

As pointed out by Mendoza et al. [14], recent observations have shown that gravitational lensing on individual [15 19], groups [20] and clusters of galaxies [21, 22] can be modelled with the standard Schwarzschild solution of general relativity, assuming the existence of a total dark plus baryonic isothermal halo, where the TullyFisher law holds for the baryonic matter. As such, the bending angle of light can be calculated using the standard lensing equation, finding that it does not depend on the impact parameter and scales with the square root of the total baryonic mass. Under a modified theory of gravity scheme with no dark matter component, the bending angle has the same value and so, since the time metric component ${ }^{(2)} g_{00}$ is known from the Tully-Fisher law as shown in equation (17), then a reconstruction of the spatial metric component ${ }^{(2)} g_{11}$ can be made. The final result is that the gravitational potentials on this modified regime of gravity have the following values:

$$
\phi=-G_{\mathrm{M}} M^{1 / 2} \ln \left(\frac{r}{r_{\star}}\right), \quad \text { and } \quad \psi=-G_{\mathrm{M}} M^{1 / 2} .
$$

Comparison of this last equation with relations (23) and (17) yields:

$$
\gamma=1
$$

We thus conclude that the relativistic structure of the underlying theory of gravity at individual, groups and 
cluster of galaxies scales is compatible with that found in the solar system, where $\gamma=1$. The difference however, lies on the fact that the gravitational potential $\phi$ appearing in equation (19) is not the one associated with Kepler's third law, but the one inferred from the TullyFisher law.

\section{DISCUSSION}

A number of independent astrophysical observations strongly support the view that the scale invariance of Newtonian gravity breaks down at scales that depend on the mass and characteristic sizes of the systems involved.

In the non-relativistic weak field limit of approximation, the behaviour of gravity is Newtonian, and the full relativistic theory that describes objects in this regime is general relativity. At the very weak field limit of approximation, sufficiently far from the masses that produce the gravitational field, Kepler's third law is modified through the Tully-Fisher law and the underlying relativistic theory in this regime is so far unknown.

We have explored some relativistic properties of this modified regime of gravity at the weak field limit of approximation, assuming that gravity is a geometrical phenomenon and that the Einstein Equivalence Principle holds. This is sufficient to build a model independent approach of the relativistic regime at second perturbation order $\mathcal{O}(2)$, in complete analogy to the one used at solar system scales where the dynamics are compatible with Einstein's general relativity. Using this modified Kepler's third law and lensing observations for individual, groups and clusters of galaxies, and interpreted in the way Mendoza et al. [14] did, we have shown that this relativistic approach is in exact compatibility with these observations. In isotropic coordinates, the non-relativistic gravitational potentials $\phi$ and $\psi$ defined in equations (12) and (23) are proportional to each other, i.e. $\phi \propto \gamma \psi$, where $\gamma$ is a Parametrised Post Newtonian Parameter in this new regime of gravity where Kepler's third law does not hold. As shown in this article, lensing observations require $\gamma=1$, as in general relativity. Note that in spherical Schwarzschild coordinates both gravitational scalar potentials differ from each other as its evident from equations (25). For the case of Einstein's general relativity these potentials are coincidentally equal not only in isotropic coordinates, but also in spherical Schwarzschild coordinates.

As shown in this article, the bending of light in regions where the Tully-Fisher law is satisfied can be predicted at second order perturbation without knowledge of the underlying relativistic theory of gravity. This is fully consistent with Einstein's view on the geometrical nature of space-time and relativistic motion. Any viable extended relativistic theory of gravity should be in agreement with the light bending predictions discussed here. The proposal by Bernal et al. 23] with lensing applications detailed in Mendoza et al. 14] is an example of such kind of theory.

\section{ACKNOWLEDGEMENTS}

This work was supported by a DGAPA-UNAM grant (IN111513-3), by the Programa de Cooperacion Cientifica UNAM-CSIC, by the Spanish grants FIS201129813-C02-02 and i-LINK0780 (CSIC), and by the JAEdoc program of the Spanish Research Council (CSIC). SM acknowledges economic support from CONACyT (26344) and the hospitality of the Instituto de Física Corpuscular (Universidad de Valencia \& CSIC). GJO acknowledges the hospitality of the Instituto de Astronomía at the Universidad Nacional Autónoma de México where the first ideas of this article were first developed.
[1] I. Newton, The Mathematical Principles of Natural Philosophy 3rd ed., The Mathematical Principles of Natural Philosophy (B. Motte, 1729).

[2] J. Kepler, C. Ptolemaeus, and R. Fludd, Lincii Austriae, sumptibus G. Tampachii, excudebat I. Plancvs, 1619. (1619).

[3] B. Famaey and S. S. McGaugh, Living Reviews in Relativity 15, 10 (2012), arXiv:1112.3960 [astro-ph.CO].

[4] X. Hernandez, S. Mendoza, T. Suarez, and T. Bernal, Astronomy and Astrophysics 514, A101 (2010), arXiv:0904.1434 [astro-ph.GA]

[5] X. Hernandez and M. Jiménez, Astrophys. J. 750, 9 (2012) arXiv:1108.4021 [astro-ph.CO].

[6] X. Hernandez, M. A. Jiménez, and C. Allen, MNRAS 428, 3196 (2013), arXiv:1206.5024 [astro-ph.GA]
X. Hernandez, M. A. Jiménez, and C. Allen, European Physical Journal C 72, 1884 (2012), arXiv:1105.1873 [astro-ph.GA]

[8] M. Milgrom, Astrophys. J. 270, 365 (1983)

[9] M. Milgrom, in EAS Publications Series, EAS Publications Series, Vol. 20, edited by G. A. Mamon, F. Combes, C. Deffayet, and B. Fort (2006) pp. 217224, arXiv:astro-ph/0510117.

[10] C. M. Will, Theory and Experiment in Gravitational Physics, by Clifford M. Will, pp. 396. ISBN 0521439736. Cambridge, UK: Cambridge University Press, March 1993. (Cambridge University Press, 1993).

[11] C. M. Will, Living Reviews in Relativity 9, 3 (2006), arXiv:0510072 [gr-qc],

[12] L. Landau and E. Lifshitz, The classical theory of fields, Course of theoretical physics (Butterworth Heinemann, 1975).

[13] C. Misner, K. Thorne, and J. Wheeler, Gravitation, Physics Series (W. H. Freeman, 1973). 
[14] S. Mendoza, T. Bernal, X. Hernandez, J. C. Hidalgo, and L. A. Torres, MNRAS 433, 1802 (2013), arXiv:1208.6241 [astro-ph.CO],

[15] R. Gavazzi, T. Treu, J. D. Rhodes, L. V. E. Koopmans, A. S. Bolton, S. Burles, R. J. Massey, and L. A. Moustakas, Astrophys. J. 667, 176 (2007), arXiv:astro-ph/0701589

[16] L. V. E. Koopmans, T. Treu, A. S. Bolton, S. Burles, and L. A. Moustakas, Astrophys. J. 649, 599 (2006), arXiv:astro-ph/0601628.

[17] M. Barnabè, O. Czoske, L. V. E. Koopmans, T. Treu, and A. S. Bolton, MNRAS 415, 2215 (2011) arXiv:1102.2261 [astro-ph.CO],

[18] S. H. Suyu, S. W. Hensel, J. P. McKean, C. D. Fassnacht, T. Treu, A. Halkola, M. Norbury, N. Jackson, P. Schneider, D. Thompson, M. W. Auger, L. V. E. Koopmans, and K. Matthews, Astrophys. J. 750, 10 (2012) arXiv:1110.2536 [astro-ph.CO],

[19] A. A. Dutton, B. J. Brewer, P. J. Marshall, M. W. Auger, T. Treu, D. C. Koo, A. S. Bolton, B. P. Holden, and L. V. E. Koopmans, MNRAS 417, 1621 (2011) arXiv:1101.1622 [astro-ph.CO].

[20] A. More, R. Cabanac, S. More, C. Alard, M. Limousin, J.-P. Kneib, R. Gavazzi, and V. Motta, Astrophys. J. 749, 38 (2012), arXiv:1109.1821 [astro-ph.CO]

[21] A. B. Newman, T. Treu, R. S. Ellis, D. J. Sand, J. Richard, P. J. Marshall, P. Capak, and S. Miyazaki, Astrophys. J. 706, 1078 (2009) arXiv:0909.3527 [astro-ph.CO]

[22] M. Limousin, J. Richard, E. Jullo, J.-P. Kneib, B. Fort, G. Soucail, Á. Elíasdóttir, P. Natarajan, R. S. Ellis, I. Smail, O. Czoske, G. P. Smith, P. Hudelot, S. Bardeau, H. Ebeling, E. Egami, and K. K. Knudsen, Astrophys. J. 668, 643 (2007), arXiv:astro-ph/0612165,

[23] T. Bernal, S. Capozziello, J. C. Hidalgo, and S. Mendoza, Eur. Phys. J. C 71, 1794 (2011) arXiv:1108.5588 [astro-ph.CO] 